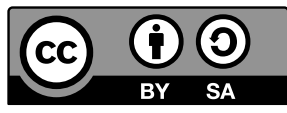

\title{
LES MÉTAPHORES SYNESTHÉSIQUES ET LE SON
}

\section{INTRODUCTION}

Pour décrire la musique et son aspect créatif et perceptif on doit, à cause du manque de termes linguistiques appropriés, recourir aux métaphores. Plus exactement, c'est la métaphore conceptuelle qui nous permet de saisir un phénomène abstrait tel que la musique et de le rendre plus sensible et compréhensible - on parle ainsi du mouvement musical, de passages, de phrases et de sons clairs ou aigus etc. Ce processus métaphorique consiste à décrire certains aspects auditifs à l'aide des termes d'un autre domaine expérientiel, $\mathrm{p}$. ex. des qualités visuelles de l'espace telles que la hauteur, la profondeur, le volume etc. sont transportées dans le domaine acoustique pour décrire l'esthétique du son.

La musique ne se déroule pas uniquement dans la sphère auditive, mais elle est un phénomène au moins bimodal ( $\mathrm{p}$. ex. le rythme comporte à la fois la dimension auditive et kinesthésique). Étant donné que l'expérience musicale implique alors le corps tout entier et que celui-ci fonctionne dans sa totalité psychophysique (l'unité du corps), notre conceptualisation et la description de la musique relèvent de cette expérience corporelle, qui n'est pas liée exclusivement au domaine auditif mais recouvre aussi d'autres domaines sensoriels. Au niveau de la langue, cette communication entre les sens est connue sous le terme synesthésie (du grec syn - union - et aisthesis - sensation), issu du lexique psychologique et médical où il désigne un phénomène cognitif qui consiste en fusion de sens différents. Compte tenu de ce croisement sensoriel basé sur des lexèmes appartenant à deux champs perceptifs, la synesthésie linguistique est souvent perçue comme un type de métaphore (conceptuelle) - on parle des métaphores synesthésiques. ${ }^{1}$ Cette transposition sensorielle qui nous permet d'embrasser la complexité de l'expérience perceptive et corporelle est répandue dans le langage usuel, mais souvent passe inaperçue - p. ex. couleur chaude, son aigu, odeur piquante, voix claire etc.

Le sujet de ce travail porte sur les métaphores synesthésiques en slovène et en français qui servent à décrire les perceptions acoustiques, plus exactement la musique. À partir des données tirées de critiques musicales on proposera une analyse de champs sensoriels

1 Les chercheurs ne sont pas unanimes sur la dénomination, c'est pourquoi il existe un bon nombre de termes, utilisés pour décrire ce phénomène linguistique de l'intégration des sens (p. ex. synesthésie, synesthésie linguistique, synesthésie-trope etc.). 
faisant partie de la description synesthésique du domaine auditif. On tâchera d'étudier les lexèmes synesthésiques employés et les qualités acoustiques qu'ils désignent, la nature lexicalisée ou vive des mots et leur valeur connotative. Une étude contrastive nous permettra de répondre à la question de l'universalité et du fondement expérientiel des syntagmes synesthésiques.

\section{THÉORIE : LA LINGUISTIQUE COGNITIVE, LA PERCEPTION SENSORIELLE ET LA SYNESTHÉSIE}

C'est la linguistique cognitive qui représente le cadre méthodologique de notre travail. Du point de vue cognitif, le langage, considéré comme une partie importante du système cognitif, se fonde sur nos expériences physiques, psychiques et socio-culturelles et reflète notre perception du monde et nos structures conceptuelles. La philosophie expérientaliste (expérientalisme), qui favorise l'idée du langage « incarné » (ang. embodied), c.-à-d. ancré dans nos expériences corporelles du soi et de l'environnement, constitue le fondement philosophique de la linguistique cognitive (Będkowska-Kopczyk 2004: 7, 29-33; Bratož 2010: 33-39, 104; Sweetser 1993: 1, 6, 12-13; Tabakowska v Jamnik 2002: 11).

Puisque notre travail se focalise sur les expressions de la perception auditive, ce paragraphe apporte quelques notions de base de la perception sensorielle. Il y a, en général, deux façons d'envisager les sens - la conception modulaire consiste à concevoir les sens comme des unités à priori indépendantes, possédant leur propre sphère de perception ( $\mathrm{p}$. ex. la couleur est perçue par la vue) et bien délimitées, tandis que la théorie de l'unité des sens (Trstenjak 1978, Merleau-Ponty 2006, Werner 1934/2011) favorise l'idée de l'interdépendance fonctionnelle des modalités sensorielles (Cacciari 2008: 428; Legallois 2012: 4). ${ }^{2}$ Dans le cadre de la perception sensorielle il faut mentionner aussi la théorie de la hiérarchisation des domaines sensoriels distinguant les sens inférieurs (ang. lower senses) et les sens supérieurs (ang. higher senses). Cette hiérarchie reflète le rôle que jouent les sens dans notre connaissance du monde - les sens supérieurs (la vue, l'ouïe) nous transmettent plus d'informations importantes que les sens inférieurs (le goût, l'odorat, le toucher) et nous renseignent davantage sur le monde extérieur. En outre, une des classifications des sens repose sur la distance entre l'objet perçu et l'organe sensoriel - le contact physique direct est caractéristique pour les sens inférieurs (surtout le toucher et le goût), alors que les sens supérieurs ne reposent pas sur cette proximité physique. Du fait que les perceptions des sens inférieurs sont qualifiées comme plus directes et concrètes, on leur accorde une valeur subjective et intime, contrairement aux perceptions des sens supérieurs qui sont perçus comme indirectes, abstraites et sont liées plutôt à l'objectivité et à la raison (Shen, Aiseman 2008: 112; Sweetser 1993: 38-44).

2 La conception modulaire des sens considère la synesthésie comme une anomalie, tandis que dans la théorie de l'unité des sens, la synesthésie est un phénomène perceptuel normal. 
On connaît deux types de synesthésie - la synesthésie en tant que phénomène neurologique (synesthésie perceptuelle) et la synesthésie linguistique. Bien que les deux types de synesthésie divergent sur plusieurs plans, ils connaissent aussi certains points communs quant à des corrélations établies, p. ex. dans les deux cas les qualités optiques (notamment la clarté) sont projetées sur le domaine auditif (la hauteur du son). Les chercheurs ne s'accordent pas sur le rapport entre la synesthésie perceptuelle et linguistique d'après certains le lien entre les deux phénomènes existe, d'autres, par contre, s'inscrivent en faux contre 1'existence de cette liason (Day 1996: 12; Szántó 2011: 9-11; Legallois 2004: 5; Marks 2011: 56-69; Yu 2012: 1284-1285). De même, il n'y a pas d'explication unanime en ce qui concerne la relation entre la synesthésie linguistique et la métaphore. Ces constructions lexicalisées ou vives dont le rôle discursif consiste soit à évaluer soit à désigner les perceptions sensorielles, sont le plus souvent considérées comme un type spécial de métaphore. La majorité des théories envisage le phénomène dans une perspective cognitive comme une métaphore conceptuelle qui consiste en un croisement de deux modalités sensorielles servant du domaine source et du domaine cible. Pareillement, quelques-uns les traitent comme des métaphores universelles primaires se formant très tôt de manière intuitive et subconsciente et se basant sur l'expérience (Bretones Callejas 2001: 3; Day 1996: 1, 14; Seizt 2005: 1-3; Szántó 2011: 9-11). Cependant, il reste à déterminer le fondement sur lequel s'établit l'analogie entre les modalités sensorielles. ${ }^{3}$ Les transferts entre les domaines sensoriels connaissent leur propre structure intérieure démontrée par le principe de la directionalité (Day 1996, Sadamitsu 2004, Ullmann 1957, Williams 1976 etc.). La répartition des sens va des sens inférieurs aux sens supérieurs : le toucher - le goût - l'odorat - la vue/l'oü̈e. C'est le champ auditif qui sert du domaine cible le plus fréquent tandis que les perceptions tactiles représentent le domaine source le plus courant. Les sens s'organisent par égard à leur rôle dans le système cognitif ou en considération de leur degré du concret (c.-à-d. de l'accessibilité). Ce dernier (le degré du concret) tient à trois facteurs : le contact direct entre la stimulation sensorielle et l'organe sensoriel, l'absence d'un organe sensoriel specialisé et la possibilité de déterminer la source de la stimulation sensorielle. Étant donné que le son n'a pas d'existence spatiale visible (de même que l'odeur), il est qualifié comme abstrait et décrit en conséquence en termes de propriétés/modalités sensorielles ayant une dimension spatiale (Cacciari 1998: 128-129; Day 1996: 6; Sadamitsu 2004: 117; Shen, Aiseman 2008: 112).

3 Ces transpositions sensorielles peuvent dériver de nos propres expériences concernant la formation du son (les sons aigus résonnent dans la tête tandis que les sons graves résonnent dans le bas du corps ou dans la poitrine) ou bien elles reflètent les lois de $1^{\prime}$ environnement physique (les objets larges sonnent avec des fréquences basses - les sons graves sont décrits comme amples). Parmi les explications répandues figurent aussi la théorie de 1'association qui attribue ces corrélations à la cooccurrence de deux expériences et la théorie du retentissement affectif qui insiste sur le rôle de la réaction subjective (similaire) dans la transposition sensorielle (c.-à-d. deux expériences partagent la même dimension émotionnelle) (Cox 2001: 195, 203; Marks 2011: 55; Segalen 1902: 4; Shibuya, Nozawa 2003: 408-413, Werner 1934/2011: 167). 


\section{3} MÉTHODOLOGIE

Méthodologie. Sur le plan méthodologique on se sert de l'approche textuelle, lexicographique, contrastive et de l'analyse de corpus monolingue. L'approche textuelle repose sur l'examen de 346 critiques musicales en français et en slovène. Elles offrent une description globale de l'impression auditive, qui est à la fois subjective et objective, en plus, les lexèmes utilisés sont aussi bien lexicalisés que vifs. L'avantage de l'approche proposée sont les donnés authentiques qui révèlent la langue en usage et ses tendances synesthésiques. Dans la suite, on dresse un tableau des sources slovènes et françaises des critiques musicales:

\section{Les sources slovènes des critiques musicales:}

- médias de presse (versions papier et électronique): Delo (10) ${ }^{4}$, Dnevnik (5), Mladina (20);

- $\quad$ médias électroniques: Radio Študent (35), RTVSLO (10), Pogledi.si (6) ;

- médias musicaux électroniques: Odzven (10), RockLine (10), Rockonnet (10), Nova Muska (16);

- revue musicale (version papier): Glasna (20).

\section{Les sources françaises des critiques musicales:}

- médias de presse (versions papier et électronique): Le Monde (10), Libération (10), Le Nouvel Observateur (12), Les Inrocks (40), Marie Claire (9);

- médias de presse musicaux (versions papier et électronique): Rock\&Folk (20);

- revue musicale (version papier): Classica (43);

- médias musicaux électroniques: Citizen Jazz (20), Millefeuille (10), Foutraque (10), Le Cargo (10).

Outre l'approche textuelle, on emploie aussi la méthode lexicographique (SSKJ, TLFi, Francosko-slovenski slovar) pour l'étude sémantique de termes synesthésiques lexicalisés, et l'analyse de corpus monolingue (korpus Kres - uniquement pour des données slovènes) au moyen de laquelle on examine la fréquence de lexèmes synesthésiques lexicalisés. Reste l'approche contrastive qui est indispensable pour la recherche de similitudes et de divergences au niveau de la description synesthésique des sensations auditives.

Choix de lèxemes et (sub)divison des catégories sensorielles. Notre base de données linguistiques se compose de lexèmes ayant le sens sensoriel et appartenant aux domaines du goût, du toucher, de la vue et de l'odorat. Les classes de mots prises en considération sont l'adjectif, le substantif, le verbe, et l'adverbe. En ce qui concerne la catégorisation sensorielle des données, ce sont des classifications connues, par exemple

4 Le nombre de critiques analisées. 
celles de Williams (1976), d'Ullmann (1957), de Day (1996) etc., qui nous servent de modèle (avec quelques modifications).

Le classement de base se fait au niveau de quatre sens qui représentent les domaines sources. Les lexèmes représentant les sensations olfactives ne sont pas additionnellement classifiés, alors que le champ du goût est divisé en cinq groupes : " sucré », " amer », " sale », « aigre » et « (non)-délicieux ». Les lexèmes de la perception visuelle sont classés en quatre groupes : « sombre, clair »- les lexèmes désignent le degré de la clarté lumineuse, « transparence, visibilité » - leur sens commun rélève du degré de transparence et de visibilité, « couleur»-les lexèmes dénotant la couleur et la création en art plastique et « dimension »- le dénominateur commun est celui de la dimension ${ }^{5}$. Les termes appartenant au champ sensoriel du toucher sont sur le premier plan divisés en trois groupes par égard à la façon dont on perçoit la stimulation tactile : « perception manuelle (haptique)», « perception cutanée » et « perception corporelle ». Le sens haptique implique l'exploration manuelle active d'objets qui, de même que la vue, sert à apprécier l'espace. Puisque la perception tactile est graduelle, intentionnelle et consciente elle est considérée comme subjective et affective. Le groupe est subdivisé en trois catégories : « structure », « surface (texture) », « perception tactile ». La première catégorie (« structure ») se compose de deux sous-domaines - « résistance à la pression » comporte des lexèmes désignant le degré de la dureté, de la souplesse et de la densité, tandis que dans « décomposition et formation " sont inclus les lexèmes qui indiquent la division d'un tout en ses parties simples sous l'influence de la pression ou des forces extérieures. La deuxième catégorie ( « surface ») consiste en deux sous-domaines - « poli » et « tranchant »-qui se réfèrent au degré soit de la rugosité soit du tranchant, alors que les lexèmes de la dernière catégorie («perception tactile») expriment le degré de l'intensité du toucher. La perception cutanée passive nous permet de sentir (entre autres) la température et l'humidité, qui sont aussi ses sous-domaines, tandis que la perception corporelle, également passive, est formée de trois sous-domaines - « poids », « force » et « autre » (ce dernier se compose de lexèmes exprimant l'idée de la vibration ou de la tension musculaire).

5 Les lexèmes de la dimension décrivent les propriétés perceptives qui sont par leur nature synesthéstiques, parce qu'on peut les percevoir avec le toucher et la vue. 
Tableau 1: Catégories sensorielles

\begin{tabular}{|c|c|}
\hline SENS & CATÉGORIES SENSORIELLES AVEC LES EXEMPLES \\
\hline Odeur & smrdeti, odeur \\
\hline Goût & $\begin{array}{l}\text { Sucré : medeno, sucrerie } \\
\text { Amer : grenek, amer } \\
\text { Sale : posoljen } \\
\text { Aigre: acide } \\
\text { (Non)-délicieux : sočno, revêche }\end{array}$ \\
\hline Vue & $\begin{array}{l}\text { Sombre, clair : iskriv, lumière } \\
\text { Transparence, visibilité : jasnost, diaphane } \\
\text { Couleur : pisano, nuance } \\
\text { Dimension : globina, épaisseur }\end{array}$ \\
\hline Toucher & $\begin{array}{l}\text { Perception manuelle: } \\
\quad \text { Structure : } \\
\quad \text { Résistance à la pression : mehak, dureté } \\
\text { - Surface (texture): } \\
\quad \text { Poli : žameten, rugosité } \\
\quad \text { Tranchant : oster, rondelet } \\
\text { - Perception tactile : tipati, caresser } \\
\text { Perception cutanée: } \\
\text { - Température : hladnost, ardent } \\
\text { - Humidité : sveže, sécheresse } \\
\text { Perception corporelle: } \\
\text { - Poids : lahek, lourdeur } \\
\text { - Force : močen, frêle } \\
\text { - Autre : vibracija, palpitant }\end{array}$ \\
\hline
\end{tabular}

\section{$4 \quad$ PARTIE ANALITIQUE}

L'analyse se focalise dans un premier temps sur la répartition et la fréquence des domaines sensoriels et des classes de mots, suit la présentation des mots lexicaux et des familles de mots ayant le sens sensoriel, ensuite on examine le sens sensoriel de mots synesthésiques et leur nature lexicalisée et dans un dernier temps on se penche sur la valeur connotative de termes sensoriels proposés.

\section{1 Hypothèses}

Notre intérêt primordial est d'examiner la façon dont on décrit la complexité de l'impression auditive dans les critiques musicales avec les moyens linguistiques tels que la synesthésie. La question substantielle de l'analyse contrastive franco-slovène concerne le rapport entre l'universalité (la nature) et la dimension socio-culturelle (la culture) des combinaisons synesthésiques. Compte tenu de notre cadre méthodologique qui est celui 
de la linguistique cognitive ${ }^{6}$ (la sémantique cognitive) et qui présuppose la motivation expérientielle (corporelle, physique, psychologique, socio-culturelle etc.) des métaphores (ang. embodiment) on se penche sur la thèse selon laquelle les constructions synesthésiques reposent sur nos expériences perceptives universelles et se développent sous l'influence du contexte socio-culturel. Si le rapport entre la langue et la perception sensorielle existe il devrait se manifester par des parallélismes et des concordances au niveau de la description synesthésique (y compris la valeur connotative) des sensations acoustiques. On suppose d'ailleurs que les divergences entre les langues sont rares étant donné que notre recherche est basée sur des langues qui émanent du contexte socio-culturel similaire (la société de l'Europe occidentale).

On présume aussi que la répartition des classes sensorielles qui servent du domaine source est identique dans les deux langues et qu'elle obéit au principe de la directionalité des transferts, c.-à-d. que la transposition se passe des sens inférieurs (le toucher, l'odorat, le goût) aux sens supérieurs (l'oüe, la vue). A partir de ce principe, on présuppose que la modalité sensorielle prévalente dans la description synesthésique est le toucher. On pense aussi que la classe de mots prédominante est l'adjectif car il permet de décrire les impressions acoustiques de façon concrète et expressive.

De plus, on suppose que les lexèmes appartenant aux sens inférieurs sont, comparé à ceux des sens supérieurs, plus fréquemment dotés de la dimension évaluative. Cela s'explique par le fait que les sens inférieurs sont en raison du contact physique direct entre un organe sensoriel et un objet de la perception considérés comme plus concrets et par conséquent subjectifs, tandis que les sens supérieurs ne reposent pas sur cette proximité physique et sont par conséquent perçus comme plus objectifs.

Parmi nos hypothèses majeures figure aussi celle selon laquelle la proportion de termes ayant une dimension connotative et ceux qui sont neutres est équilibrée. Cette supposition tient à la source de nos données - la critique musicale - dont le rôle consiste à décrire et évaluer l'impression auditive et pour ce faire elle combine à la fois la description subjective et objective.

\section{2 Analyse quantitative}

On propose une analyse quantitative des données slovènes et françaises examinant l'ordre des classes sensorielles qui servent de domaine source et la fréquence des lexèmes synesthésiques par rapport à leur classe de mots.

Les résultats de l'analyse démontrent que, dans les deux langues, ce sont les perceptions tactiles qui servent du domaine source le plus fréquent, cependant, une grande partie des perceptions auditives est décrite en termes du champ visuel. Beaucoup plus rares sont

6 Du fait que la linguistique cognitive favorise l'idée de l'unité du corps, de l'environnement et de la cognition elle fournit une base pertinente pour notre recherche de ce phénomène linguistique. 
les projections des perceptions gustatives et olfactives sur la modalité auditive.

Cet ordre des classes sensorielles peut s'expliquer par deux aspects. Le premier touche le rôle des sens dans notre vie quotidienne - la vue est le sens prévalent étant donné qu'il est omniprésent et nous livre les informations les plus importantes du monde extérieur, ce qui ne vaut pas pour le goût et l'odorat auxquels on accorde moins d'importance dans notre interaction avec le monde. L'autre explication repose sur le degré de l'accessibilité des sens qui sont perçus comme plus ou moins abstraits/concrets et ce sont notamment les sens qualifiés de concrets qui servent le plus fréquemment du domaine source. Du fait que les perceptions auditives n'ont pas d'existence spatiale visible et sont du coup considérées comme abstraites, elles ont tendance à être caractérisées au moyen des sens inférieurs, conçus comme concrets et subjectifs. ${ }^{7} \mathrm{C}^{\prime}$ est ce qu'attestent aussi les résultats obtenus où dominent les perceptions tactiles manuelles, qui impliquent des mouvements actifs et intentionnels de la main, et c'est pourquoi les termes appartenant à la modalité tactile (manuelle) offrent la description la plus subjective de l'impression auditive. À la perception tactile (manuelle) s'ajoutent les termes du domaine visuel qui, par contre, apportent une description plus objective.

Néanmoins, la répartition des sens ne correspond pas au classement des transferts sensoriels le plus répandu, à savoir le toucher - le goût - l'odorat - la vue/l'ouïe. Dans notre cas la classificaction est la suivante : le toucher - la vue - le goût - l'odorat (le pourcentage des perceptions visuelles et tactiles est presque égal). À cet égard, il faut prendre en considération que le choix et la répartition des termes synesthésiques sont assez relatifs et issus du jugement subjectif. Du reste, une partie considérable des lexèmes dénote des qualités qui sont synesthésiques de nature (les qualités spatiales), ce qui veut dire qu'on peut les percevoir par divers sens (notamment le toucher et la vue).

Quant à la fréquence des classes de mots, comme prévu, dans les deux langues prévalent les adjectifs auxquels succèdent les substantifs, les verbes et les adverbes.

Tableau 2: Répartition des lexèmes synesthésiques slovènes

\begin{tabular}{|c|c|c|c|c|c|c|}
\hline & Adjectif & Substantif & Verbe & Adverbe & Total & \\
\hline Ouïe & 2 & 1 & 2 & 2 & 7 & $(1 \%)$ \\
\hline Goût & 23 & 8 & 4 & 5 & 40 & (7\%) \\
\hline Vue & 133 & 86 & 34 & 12 & 265 & (45\%) \\
\hline Toucher & 149 & 82 & 29 & 19 & 279 & (47\%) \\
\hline Total & $307 \quad(52 \%)$ & $177 \quad(30 \%)$ & $(12 \%)$ & (6\%) & 591 & \\
\hline
\end{tabular}

7 Les facteurs qui conditionnent la nature abstraite ou concrète des sens sont présentés dans le chapitre théorique. 
Tableau 3: Répartition des lexèmes synesthésiques français

\begin{tabular}{|c|c|c|c|c|c|c|}
\hline & Adjectif & Substantif & Verbe & Adverbe & Total & \\
\hline Ouïe & - & 5 & 3 & - & 8 & (1\%) \\
\hline Goût & 36 & 24 & 8 & 5 & 51 & $(7 \%)$ \\
\hline Vue & 131 & 121 & 32 & - & 284 & (38\%) \\
\hline Toucher & 231 & 145 & 30 & 1 & 407 & (54\%) \\
\hline Total & $398 \quad(53 \%)$ & (39\%) & $(10 \%)$ & $(1 \%)$ & 750 & \\
\hline
\end{tabular}

\section{3 Analyse qualitative et quantitative}

À présent, nous allons présenter les lexèmes et les familles de mots synesthésiques les plus fréquents. ${ }^{8}$ Dans la plupart des cas on peut établir un parallèle entre les données françaises et slovènes.

Les lexèmes désignant les perceptions olfactives sont les plus rares et il n'y a que le substantif odeur qui apparaît trois fois.

Dans le domaine gustatif, prévalent les lexèmes exprimant le goût sucré (sladeksucré), en plus, en français, ce sont aussi les lexèmes rélatifs à la saveur acide (acide) qui sont courants.

Parmi les lexèmes de la perception visuelle prévale la paire barva - couleur, il y a encore d'autres qui proviennent du champ sémantique de la luminosité (svetel - lumineux et zablesteti - briller), de la transparence (jasen - clair) ou de la dimension spatiale ( $\mathrm{glo}$ bok - profond, masiven - épais in širina - ample). Parmi les lexèmes slovènes les plus courants figure aussi le couple čist-umazan.

Les lexèmes du champ tactile prédominants désignent le degré de la dureté (trdotadureté), de la solidité ou de la densité (čvrst-ferme), de la souplesse (prožen - souple) et de la mollesse ou fragilité (mehek - délicat). Ils signifient aussi la division d'un tout en ses parties simples sous l'influence de la pression ou des forces extérieures (lomljenrompre). Dans les deux langues, les plus fréquents sont les lexèmes dénotant le tranchant (oster - aigu) ou la rugosité (raskav - rugueux). Puis, il y a aussi des lexèmes du champ sémantique de la température qui marquent divers degrés thermiques : une grande chaleur (žgati - brûler), la chaleur (topel - chaud), la tiédeur (mlačnost) et le froid (hladen, mr$z e l$-froideur). En outre, ce qui est assez fréquent, notamment en français, c'est la paire décrivant la sécheresse $(s u h-s e c)$. Dans le champ sémantique du poids prévalent les lexèmes dénotant la lourdeur (težek - grave) ou la légèreté (lahkoten-léger), tandis que l'idée de la force, aussi courante, est exprimée par le couple močen - puissant.

8 Plus précisément, on se concentre sur ceux qui apparaissent au moins trois fois. 


\section{4 Analyse lexicale (sens synesthésiques lexicalisés)}

Pour étudier les sens synesthésiques lexicalisés, on se sert de la méthode lexicographique (SSKJ, TLFi, Francoski-slovenski slovar) et de l'analyse de corpus (korpus Kres). Avec l'analyse lexicale on examine quel est le sens sensoriel de mots synesthésiques lexicalisés et on vérifie nos hypothèses selon lesquelles la proportion entre les lexèmes synesthésiques lexicalisés et vifs est équilibrée et la dimension connotative des lexèmes synesthésiques lexicalisés est neutre.

Les résultats de notre analyse montrent que les lexèmes ayant le sens synesthésique lexicalisé désignent les caractéristiques principales du son (la hauteur, l'intensité et le timbre du son), ils peuvent décrire l'intensité de l'impression auditive (cette intensité ne touche pas la puissance du son mais d'autres qualités auditives) ou bien ils apportent une dimension affective (agréable ou désagréable aux sens), qui dans la plupart des cas résulte du degré de l'intensité.

Le ton haut est dénoté par les lexèmes suivants : acide, acidulé, aigrelet (la saveur acide), svetel - clair (la luminosité), visok - haut (la hauteur), tanek (la minceur), aigu, acéré, tranchant (le tranchant), sec (la sécheresse) et léger (la légèreté), p. ex. "[T]anjšsi, višje uglašeni par bobnov » (Vidmar 2015). Par contre, le ton bas caractérisent les lexèmes : temen, sombre, obscurcir (l'obscurité), globok - profond, nizek (le bas-fond), debel - gros (l'épaisseur) et težek - lourd, grave (la lourdeur), p. ex. "[L]a voix grave et rauque » (Desassis 2016).

Pour qualifier la clarté et la netteté sonore on utilise les lexèmes svetel - clair (la luminosité), jasen, čist - pur, limpide (la transparence), sec (la sécheresse) et svež (la fraîcheur), p. ex. "[U]ne voix claire et chaleureuse » (Roux 2015), tandis que pour un son qui n'est pas clair et net on se sert des termes obscurcir, temen - sombre (l'obscurité), brumeux, terne (la transparence), globok - profond, nizek (le bas-fond), debel-gros (l'épaisseur), raskav, hrapav - râpeux, rocailleux, éraillé (la rugosité) et težek - lourd (la lourdeur), p. ex. "[Z]elo zrel zvok, ki se giblje med temačno hladnostjo alter rocka » (Njegovan 2015). Les expressions synesthésiques dénotant un ton haut expriment d'habitude aussi l'idée de la clarté sonore (svetel - clair), au contraire, celles qui désignent un ton bas servent aussi à qualifier le manque de clarté sonore (temen - sombre), p. ex. « $[P]$ evka s korajžnim temnikasto hrapavim glasom » (Mehle 2015).

Le timbre est exprimé par la paire barva-couleur, les termes sočen (le goût) et svež (la fraîcheur) dénotent la belle sonorité d'une voix, de même, le couple žameten - velours (le poli) est lié à l'euphonie, alors que les lexèmes rond (la rondeur), volume et ample (l'ampleur) s'appliquent à la force et à la plénitude de l'impression auditive, p. ex. " [L] a sonorité, ronde et chaleureuse » (Bonnaure 2014b).

Pour caractériser une grande intensité et une manifestation prononcée d'un phénomène auditif, on emploie les termes surov - cru (le goût), brillant (la luminosité), haut (la hauteur), ample (l'ampleur), gros (l'épaisseur), trd - dureté, âpre, trden, čvrst (la résistance à la pression), oster - acéré, aigu (le tranchant), rond (la rondeur), sec (la 
sécheresse), lourd (la lourdeur) et renforcé (la force), p. ex. " [V]se tja do ostrih, s punkovskim robom odigranih komadov » (Batelić 2016). Dans le cas où la grande intensité évoque plus de force, de sonnorité ou de solidité, elle a une connotation positive (p. ex. rond, brillant, ample, trden, čvrst, renforcé), p. ex. "[O]rkester s čvrstim in homogenim zvenom » (Vučko 2015), par contre une grande intensité est connotée négativement quand elle dénote la rudesse, le manque de sensibilité ou de douceur et la sonorité moins agréable (p. ex. âpre, dureté, acéré, sec), p. ex. "[L]es cordes un peu sèches manquent d'épaisseur » (Fleury 2014). Un faible degré de l'intensité et une manifestation sonore moins accentuée sont qualifiés par les adjectifs doux (le goût sucré), medel (le manque de luminosité), terne, brumeux (le manque de transparence), nežen, mehek, krhek - tendre, délicat, moelleux (le manque de résistance à la pression), léger (la légèreté) et šibek, slaboten - frêle (le manque de force), p. ex. " [O]bčinstvo je same mehke, skoraj neslišne prehode med skladbami prekrilo z nepotrebnim vmesnim aplavzom » (Stolič 2015). De plus, l'intensité faible est souvent liée à ce qui est agréable et bienfaisant, du coup, sa dimension connotative est positive (doux, nežen, mehek, tendre, délicat, moelleux etc.), p. ex. " [M]ehak, topel analogen lo-fi zvok» (Katarina J. 2015).

Pour la description des changements de l'intensité et de la hauteur sonore, du timbre, du rythme etc. on emploie les termes nuancer (la couleur), amplifier, élever (l'ampleur), lomiti - briser, trgati (la décomposition), vibracija - vibrer et trepetati - tremblant (le tremblement), p. ex. "[L]ignes mélodiques se brisent » (Dourlhès 2016).

Une partie considérable des lexèmes servent surtout à évaluer un phénomène auditif. Cette valeur affective est positive si les termes désignent ce qui est beau, agréable (aux sens), vif, euphonique, harmonieux etc. (sladek - sucré, suave (le goût sucré), topel chaleur, chaud (la chaleur), frais (la frâicheur) etc., p. ex. " [P]ianist je navdušil s toplim tonom » (Vučko 2016), et négative s'il s'agit d'une stimulation auditive désagréable, dissonante ou peu harmonieuse (osladen - doucereux (le goût sucré), rugueux (la rugosité), hladen, mrzel - froideur (la froideur) et suh - sec (la sécheresse) etc.), p. ex. «[D]u rock'n'roll au coeur brisé, sentimental, mais jamais doucereux » (Farkas 2014).

On peut confirmer notre première hypothèse selon laquelle la proportion entre les lexèmes synesthésiques lexicalisés et vifs est dans une large mesure équilibrée. Il en va de même pour la deuxième hypothèse adoptée qui concerne la valeur connotative des lexèmes. Ceux-ci sont en majeure partie neutres, quelques-uns ont une connotation positive (svetel, sočen, svež, žameten ; suave, doux, dessiné, délicat, moelleux, soyeux, velours), alors que certains sont connotés négativement (acidulé, revêche, âpre, rugueux).

\section{5 Analyse de la valeur connotative}

Dans l'analyse de la valeur connotative, on se concentre sur les hypotèses que la proportion entre les lexèmes synesthésiques neutres et connotés est équilibrée et que la valeur 
connotative est plus fréquente pour les lexèmes synesthésiques vifs et pour les sens inférieurs.

Les lexèmes de la perception olfactive sont dans la plupart des cas connotés - leur connotation est positive s'ils expriment une odeur agréable (dehteti, fleurer), p. ex. « [D] es compositions qui fleurent bon le blues » (Dussutour 2016), et négative dans le cas où l'odeur est qualifiée de mauvaise (smrdeti, postano, un relent), p. ex. "[V]se skupaj zveni bolj postano » (Borka 2015a).

La valeur connotative est ajoutée aussi aux lexèmes dénotant le domaine gustatif. Ceux-ci sont connotés positivement quand ils désignent un goût sucré (sladek-doux) et agréable (okusen - délicieux), p. ex. " [N]advse okusne kitarsko-klavirske harmonije » (P. Podbrežnik 2016), et négativement quand ils décrivent un goût désagréable (trpekrêveche), trop intense et peu naturel (osladen - douceureux) ou même le manque de goût, p. ex. " [O]verdose osladnih kitarskih rifov » (Borka 2015b). La valeur neutre caractérise les lexèmes qualifiant le degré d'intensité d'un phénomène acoustique (acide, douceur) ou le goût en général (goût), p. ex. "[L]es morceaux /.../ alliant douceur extrême et force » (Gab 2014).

Les perceptions visuelles sont pour la plupart connotées, mais il faut prendre en considération que la frontière entre la valeur neutre et connotée des lexèmes n'est pas nette, mais plutôt rélative et poreuse (surtout relativement à certaines qualités visuelles - la luminosité et la transparence (svetel - clair etc.), p. ex. "[U]n ton solide et clair » (Bonnaure 2014a). Parmi les lexèmes ayant un sens neutre figurent le plus couramment les lexèmes de la dimension spatiale (globok - profond, visok), p. ex. "[V]isok, pločevinast zvok snera » (Baša 2016), moins fréquents sont ceux liés à la couleur (barvacouleur), p. ex. "[L]es couleurs de l'orchestre » (Oddon 2014). Une valeur neutre est donnée aussi aux lexèmes qui dénotent la visibilité, la luminosité ou la transparence et qui au sens figuré se réfèrent à l'ntensité de l'impression auditive (čist-limpide, netteté), p. ex. "[L]es contrastes dynamiques sont amplifiés par la netteté du jeu 》 (Venturini 2014a). Par contre, les lexèmes de la lumière vive qui au sens figuré désignent un degré plus haut de l'intensité, de la puissance ou de la vivacité d'un phénomène auditif qualifié de splendide et éclatant, ont une connotation positive (bleščeč, briller), p. ex. " [V] hitrih stavkih je lahkoten, iskriv in bleščeč » (Smrekar 2014). De même, la valeur positive est ajoutée aux lexèmes exprimant l'idée de la transparence et qui au sens figuré décrivent un son clair, pur, intense et sonore (čist, jasen-clair), p. ex. "[S]kladbe, ki so olepšane s čistim vokalom " (Novak 2016), "[L]e beau piano à la sonorité claire et chaude" (Drillon 2014). D'ailleurs, les termes qui ont des connotations positives sont aussi ceux dont le sens primaire concerne la diversité de couleurs tandis que leur sens figuré touche la variété sonore et musicale (mavričnost, barvit - coloré), p. ex. "[Z]ato je Ravelova instrumentacija /.../ zazvenela v vsej svoji mavričnosti » (Vučko 2015).

Les lexèmes portant une valeur négative sont ceux qui dénotent le manque de coloris ou métaphoriquement de variété sonore (monochrome), p. ex. "[L]>absence de 
variations de couleurs /.../ fait que l'interprétation tourne à vide " (Friédérich 2014) et ceux désignant le manque de luminosité et la visibilité réduite ou figurément le manque de clarté et l'intensité sonore faible (medel, brouillard), p. ex. " [U]n concert noyé dans un épouvantable brouillard sonore »(Venturini 2014b).

Parmi les lexèmes de la perception tactile prévalent ceux qui sont neutres et qui décrivent l'intensité d'une impression auditive. Cependant, de même que dans le domaine visuel, la frontière séparant les lexèmes connotés de ceux qui sont neutres est floue et donc souvent difficile à déterminer. Parmi les lexèmes neutres figurent ceux dénotant l'intensité qui est soit grande et se révèle comme un son tranchant, puissant et clair (oster-âpre), p. ex. "[O]stre kitarske linije » (Hlebec 2016), soit faible et se manifeste sous forme d'un son moins accentué, puissant et donc plus doux et agréable (mehak - délicat). Un sens textuel neutre est caractéristique des lexèmes dénotant la décomposition et la formation et au figuré les changements de la hauteur de son, de l'intensité, du rythme etc. (lomljen, drobljenje, cassure), p. ex. «[K]omad z udarnim lomljenim ritmom » (Zagoričnik 2016). Il en va de même pour les lexèmes désignant la structure et les éléments sonores (linija - ligne), l'impression auditive typique d'un genre musical (težek - lourd, mehek, trd), la voix (hrapav - râpeux) et l'idée de jouer d'un instrument (toucher), p. ex. "[T]rdorockerski riff » (P. Podbrežnik 2016), " [I]l n’y pas touché au prélude » (Roux 2015).

La connotation positive est liée aux lexèmes dénotant la température et la force, avec le sens figuré qui évoque une grande intensité, une vivacité et un éclatant et splendide phénomène sonore (vžigajoče - brûlant, močen - puissant), p. ex. "[O]dlikuje jih močen zvok» (Steblovnik 2015). La même valeur connotative apparaît aussi chez les lexèmes décrivant l'exactitude rythmique et le son plein et fort ( $\check{c} v r s t$-solide), p ex. " [O]rkester $s$ čvrstim in homogenim zvenom » (Vučko 2015), la souplesse et la légèreté (prožen souple), p. ex. "[P]rismojeni profesorji vozijo blues s fluidnostjo in prožnostjo » (Štader 2016), ou l'impression auditive agréable et souvent de faible intensité (lahek-léger), p. ex. "[T]imbre, plus léger qu'une plume » (Seban 2010).

C'est la connotation négative qui est la plus rare et qui s'associe aux lexèmes dénotant le manque de détente, de fluidité, de légèreté, de douceur et de sensibilité (trddureté), p. ex. "[P]o ritmično in zvokovno nekoliko trdem in zategnjenem začetku se je orkester sprostil » (Smrekar 2015), ou bien de force, de plénitude et de vivacité (hladen - froideur), p. ex. "[L] a froideur de l'accompagnement renforce la dureté du piano " (Friédérich 2014).

Pour finir, on passe en revue les hypothèses adoptées. L'étude des données affirme notre hypothèse selon laquelle la proportion entre les lexèmes neutres et connotés est équilibrée ce qui correspond au genre de la critique musicale basée sur la description à la fois subjective et objective d'un phénomène acoustique. Toutefois, dans des cas nombreux la frontière distinguant le sens connoté ou neutre est fluide (p. ex. temen - svetel ; umazan - čist, jasen; lahek - težek). Selon nous le rôle des lexèmes synesthésiques 
lexicalisés et vifs est double - ils servent à la fois à décrire certaines qualités auditives (c.-à-d. combler la lacune lexicale concernant les perceptions auditives) et à les évaluer comme plus ou moins agréable aux sens.

En outre, il est vrai que les lexèmes appartenant aux sens inférieurs, à savoir le toucher, le goût et l'odorat, sont plus fréquemment connotés que ceux des sens supérieurs (la vue), ce qui, d'après nous, doit son origine à nos expériences de la perception sensorielle. Il est vrai que les stimulations des sens inférieurs sont, dans notre quotidien, plus fréquemment perçues comme agréables ou désagréables aux sens, comparé aux stimulations des sens supérieurs. De plus, cette correspondance entre la valeur connotative des lexèmes et nos expériences sensorielles est révelée aussi par le fait que la connotation négative est ajoutée aux lexèmes qui dénotent eux-mêmes dans leur sens primaire quelque chose de désagréable, à l'opposé de la connotation positive qui accompagne les lexèmes avec le sens primaire désignant ce qui est agréable. Enfin, on doit rejeter l'hypothèse concernant la relation entre la valeur connotative des lexèmes et leur nature lexicalisée, étant donné qu'aussi bien les lexèmes lexicalisés que les lexèmes vifs peuvent être les deux - connotés ou neutres.

\section{5}

\section{CONCLUSION}

En conclusion, sur la base de nombreux parallélismes et concordances au niveau de la description synesthésique des sensations acoustiques (le choix de lexèmes et leur valeur connotative lexicalisée ou (con)textuelle) on suppose qu'il s'agit d'un phénomène relativement universel et que les constructions synesthésiques reposent sur nos expériences perceptives universelles et se développent sous l'influence du contexte socio-culturel. Outre la description dénotative et connotative d'un phénomè auditif il y a aussi la répartion des classses sensorielles servant de domaine source qui se ressemblent dans les deux langues. Ce sont les lexèmes de la modalité tactile qui prévalent dans la description synesthésique, suivent les lexèmes de la perception visuelle, puis ceux du goût et finalement ceux appartenant au domaine olfactif. Il est vrai aussi que la classe de mots prédominante est, comme prévu, l'adjectif et que les lexèmes désignant les perceptions des sens inférieurs sont plus fréquemment dotés d'une valeur connotative comparé aux sens supérieurs. On affirme par ailleurs l'hypothèse que la proportion de lexèmes connotés et neutres est équilibrée. 


\section{BIBLIOGRAPHIE}

1.

BĘDKOWSKA KOPCZYK, Agnieszka (2004) Jezikovna podoba negativnih čustev v slovenskem jeziku. Kognitivni pristop. Ljubljana: Študentska založba.

BRATOŽ, Silva (2010) Metafore našega časa. Koper: Fakulteta za management.

BRETONES CALLEJAS, Carmen M. (2001) Synaesthetic Metaphors in English. http:// www.ual.es/Masters/estudiosingleses/Master_en_Estudios_Ingleses/Carmen_M Bretones_Callejas_files/here.pdf (6.3.2016).

CACCIARI, Cristina (2008) Crossing the Senses in Metaphorical Language. R. W. Gibbs (ur.), The Cambridge Handbook of Metaphor and Thought. Cambridge: Cambridge University Press, 425-444.

CACCIARI, Cristina (1998) Why Do We Speak Metaphorically? Reflections on the Functions of Metaphor in Discourse and Reasoning. A. N. Katz, C. Cacciari, R. W. Gibbs, M. Turner, Figurative Language and Thought. Oxford: Oxford University Press, 119-157.

COX, Arnie (2001) The mimetic hypothesis and embodied musical meaning. Musicae Scientiae, 5/2. 195-212. http://www.escom.org/proceedings/ICMPC2000/Wed/ Cox.htm (3. 4. 2016).

DAY, Sean (1996) Synaesthesia and Synaesthetic Metaphors. Psyche, 2/32. 2-32. http:// www.daysyn.com/Day1996.pdf (6. 3. 2016).

JAMNIK, Tatjana (2002) Konceptualizacija tipnega in vidnega zaznavanja ter njena pomenska realizacija $v$ slovenskem jeziku: Diplomsko delo. Ljubljana.

LEGALLOIS, Dominique (2012) Penser les relations entre synesthésies perceptuelles et synesthésies linguistiques. La tribune internationale des langues vivantes. 64-73. http://www.crisco.unicaen.fr/IMG/pdf/Legallois_Tribune_Internationale_2_.pdf (6. 3. 2016).

LEGALLOIS, Dominique (2004) Synesthésie adjectivale, sémantique et psychologique de la forme : la transposition au coeur du lexique. L'adjectif en français et à travers les langues. Caen: P. U. C. http://www.crisco.unicaen.fr/IMG/pdf/29_LSynesthesie_adjectivale.pdf (6.3.2016).

MARKS, Lawrence E. (2011) Synesthesia, Then and Now. Intellectica, št. 55. 47-80.

MERLEAU-PONTY, Maurice (2006) Fenomenologija zaznave. Ljubljana: Študentska založba.

SADAMITSU, Miyagi (2004) Synaesthesia re-examined: An alternative treatment of smell related concepts. S. Kawakami, Y. Oba (ur.), Osaka Univ. Papers in English Linguistics, 8. 109-125. http://www.let.osaka-u.ac.jp/eigogaku/Oupel-2003-Sadamitsu.pdf (5. 5. 2016). 
SEGALEN, Victor (1902) Les Synesthésies et l'école symboliste. Mercure de France, apr.-jun. 57-90. http://gallica.bnf.fr/ark:/12148/bpt6k105516q.image.r=segalen. langFR.f000063.tableDesMatieres (6. 3. 2016).

SEITZ, Jay A. (2005) The neural, evolutionary, developmental, and bodily basis of metaphor. New Ideas in Psychology 23. 74-95. https://www.scribd.com/document/184915549/Metaphor-Creativity-Embodied-Cognition-Brain-and-Mind-UnconsciousSeitz06 (5. 5. 2016).

SHEN, Yeshayahu/Ravid EISENAMN (2008) »Heard melodies are sweet, but those unheard are sweeter« : synaesthetic metaphors and cognition. Language and Literature, 17/2. 107-121. http://www.tau.ac.i1/ yshen/publications/Heard_melodies_ are_sweet, but_th.pdf(2.2.2016).

SHIBUYA, Yoshikata/Hajime NOZAWA (2003) Constraints on Synaesthesia. Proceedings of the Twenty-Ninth Annual Meeting of the Berkeley Linguistics Society: General Session and Parasession on Phonetic Sources of Phonological Patterns: Synchronic and DiachronicExplanations. 403-414. http://journals.linguisticsociety. org/proceedings/index.php/BLS/article/viewFile/978/758 (6. 3. 2016).

SWEETSER, Eve (1993) From Etymology to Pragmatics: metaphorical and cultural aspects of semantic structure. Cambridge: Cambridge University Press.

SZÁNTÓ, Bíborka (2011) Synaesthesia. A cognitive approach. Abstract of $\mathrm{PhD}$ thesis. Babeş-Bolyai University, Faculty of Letters, Doctoral School of Hungarology. http://doctorat.ubbcluj.ro/sustinerea_publica/rezumate/2011/filologie/Salamon_Biborka_En.pdf (5. 5. 2016).

TRSTENJAK, Anton (1978) Človek in barve. Ljubljana: Univerzum.

ULLMANN, Stephen (1957) The principles of semantics. New York: Philosophical Library.

WALDENFELS, Bernhard (2002) O ritmu čutov. http://www.dlib.si/details/URN:NB$\mathrm{N}: S I: D O C-N 06 K D 4 Y K$ (15. 3. 2016).

WERNER, Heinz (1934/2011) L'unité des sens. Intellectica, št. 55. 159-170. http://intellectica.org/SiteArchives/actuels/n55/55_6_Werner.pdf (3. 4. 2016).

YU, Xiu (2012) On the Study of Synesthesia and Synesthetic Metaphor. Journal of Language Teaching and Research, 3/6. 1284-1289.

2.

GRAD, Anton (2010) Francosko-slovenski slovar. Ljubljana: DZS.

Le Trésor de la Langue Française informatisé (TLFi). http://atilf.atilf.fr/.

Kres. Korpus slovenskega jezika. http://www.korpus-kres.net/.

Slovar slovenskega knjižnega jezika (SSKJ). Ljubljana: Izdala SAZU, Založila DZS, 1970-1991. http://bos.zrc-sazu.si/sskj.html. 
3.

BAŠA, Gašper: Up In Smoke Vol. Vii: Mars Red Sky, Belzebong, Stoned Jesus. Radio Študent 13. 3. 2016. http://radiostudent.si/glasba/r\%C5\%A1-recenzija/up-in-smokevol-vii-mars-red-sky-belzebong-stoned-jesus (4. 5. 2016).

BATELIĆ, Mario: Sainkho Namtchylak: Like A Bird Or Spirit, Not A Face. Radio Študent 8. 3. 2016. http://radiostudent.si/glasba/tolpa-bumov/sainkho-namtchylak-likea-bird-or-spirit-not-a-face (4. 5. 2016).

BONNAURE, Jacques: Gabriel Fauré. Classica marec 2014a. 93.

BONNAURE, Jacques: Cesar Franck. Classica marec 2014b. 93.

BORKA, Peaches: Rub. Mladina 9. 10. 2015a. 59.

BORKA, Peaches: Ratata: Magnifique. Mladina 24. 7. 2015 b. 57.

DESSASIS, Denis: Perine Mansuy: Rainbow shell. 27. 3. 2016. http://www.citizenjazz. com/Perrine-Mansuy-3472846.html (13. 8. 2016).

DOURLHÈS, Nicolas: David Chevallier \& Le Quatuor IXI au Pannonica. 20. 3. 2016. http://www.citizenjazz.com/David-Chevallier-le-Quatuor-IXI-au-Pannonica.html (13. 8. 2016).

DRILLON, Arnaud: Ludwig van Beethoven: Sonates pour piano. Classica marec 2014. 89.

DUSSUTOUR, Laurent: Jacky Mouvillat : l'art du jazz au sommet. 13. 3. 2016. http:// www.citizenjazz.com/Jacky-Mouvillat-1-art-du-jazz-au-sommet.htm (13. 8. 2016).

FARKAS, Basile: Rock'n'roll au coeur brisé. 2014. http://www.rocknfolk.com/site/ disquedumois.php?dumo_id=4324 (13.8. 2016).

FLEURY, Michel: Une musique de Hough. Classica marec 2014. 82.

FRIÉDÉRICH, Stéphane: Ludwig van Beethoven: Concerto pour piano ${ }^{\circ} 3$. Classica marec 2014. 88.

GAB, Pagan Poetry - Concert Privé, Paris. 2. 12. 2014. http://www.lecargo.org/spip/ pagan-poetry/concert-prive-paris/article8736.html (13. 8. 2016).

HLEBEC, Nina: Hexvessel: When We Are Death. Radio Študent 13. 2. 2016. http://radiostudent.si/glasba/tolpa-bumov/hexvessel-when-we-are-death (4. 5. 2016).

J., Katarina: Mostovi do Cvetličarne. Nova Muska 1. 10. 2015. http://novamuska. org/?p=13053 (4. 5. 2016).

MEHLE, Borut: Maraton s prvakinjo. Dnevnik 18. 11. 2015. https://dnevnik. si/1042725031 (4. 5. 2016).

NJEGOVAN, Veljko: Editors: In Dream. Mladina 9. 10. 2015. 59.

NOVAK, Primož: Amorphis pripeljali finske melodije v Mostovno (2016). RockLine 4. 4. 2016. http://www.rockline.si/podrobnosti-reportaze/amorphis-pripeljali-finske-melodije-v-mostovno-2016-8825 (4. 5. 2016).

ODDON, Coline: Pascale Dusapin. Classica marec 2014. 92. 
PODBREŽNIK, Peter: IQ: Subterranea. RockLine 2. 4. 2016. http://www.rockline.si/ podrobnosti-recenzije/subterranea-1122 (4. 5. 2016).

ROUX, Marie-Aude: Dominique Vellard explore le temps élastique de la musique médiévale. Le Monde 26.-27. 7. 2015. 21.

SEBAN: Top 50 albums. Rock, electro, rap. Les Inrocks 22. 12. 2010. 102-103.

SMREKAR, Borut: Ocenjujemo: Kraljevi orkester Concertgebouw. Delo 2. 9. 2014. http://www.delo.si/kultura/ocene/ocenjujemo-kraljevi-orkester-concertgebouw. html (4. 5. 2016).

SMREKAR, Borut: Ocenjujemo: Oranžni abonma 4. Delo 17. 2. 2015. http://www.delo. si/kultura/ocene/ocenjujemo-oranzni-abonma-4.html (4. 5. 2016).

STEBLOVNIK, Sergej: Phone Box Vandals - Live In Eindhoven (Slušaj najglasnije, 2015). Nova Muska 23. 12. 2015. http://novamuska.org/? $p=13611$ (4. 5. 2016).

STOLIĆ, Tadej: Lojze Lebič: Intrada, Archiphonia, Diaphonia, Glasba/Music, Musica Concertate. Glasna avg.-sept. 2015. 33.

ŠTADER, Terens: Srčno rokanje bluesa v brezčasnem prostoru. Odzven - spletna revija o glasbi 23. 3. 2016. http://www.sigic.si/odzven/srcno-rokanje-bluesa-v-brezcasnem-prostoru (4. 5. 2016).

VENTURINI, Philippe: 33 Variations sur une Valse de Diabelli op. 12. Classica marec 2014a. 89.

VENTURINI, Philippe: Te Deum. H. 146 + Lully: Te Deum LWV. 55. Classica marec 2014b. 90.

VIDMAR, Ičo: Obred upanja in skladnosti. Dnevnik 19. 11. 2015. https://www.dnevnik. si/1042725083 (4. 5. 2016).

VUČKO, Tine: Orkester Slovenske filharmonije. Nova Muska 6. 3. 2016. http://novamuska.org/?p=13953 (4. 5. 2016).

VUČKO, Tine: Dunajski simfoniki v zlatem abonmaju. Nova Muska 1. 12. 2015. http:// novamuska.org/?p=13442 (4. 5. 2016).

ZAGORIČNIK, Luka: Saul Williams: MartyrLoserKing. Radio Študent 12. 3. 2016. http:// radiostudent.si/glasba/tolpa-bumov/saul-williams-martyrloserking (4. 5. 2016).

\section{POVZETEK}

\section{Sinestetične metafore in zvok}

Članek preučuje pojav t. i. sinestetičnih metafor, pri katerih je eno čutno področje opisano v okviru drugega (npr. svetel zvok, oster okus, sladek vonj). Cilj primerjalne raziskave, ki temelji na analizi slovenskih in francoskih glasbenih kritik, je ugotoviti, katera čutna področja sodelujejo pri (sinestetičnem) opisu slušnih zaznav oz. natančneje glasbe in kakšna je njihova zastopanost. Pri tem se preverja načelo usmerjenosti, po katerem naj bi se sinestetični prenosi vršili v smeri od 
bolj konkretnih nižjih čutov (tip, okus, voh) k abstraktnejšim višjim čutom (vid, sluh). Pomemben predmet raziskave predstavlja tudi vprašanje ustaljenosti zbranih sinestetičnih izrazov in njihove vrednotenjske zaznamovanosti. Ker tako izhodiščno kot ciljno področje sinestetičnih prenosov zastopajo izrazi čutnega zaznavanja, je relevanten izkustveni - tj. čutnozaznavni, spoznavni in družbeno-kulturni - vidik pojava, pri čemer se kot ključno zastavlja vprašanje univerzalnosti in kulturne pogojenosti sinestetičnih zvez.

Ključne besede: sinestezija, metafora, čutno zaznavanje, glasba, zvok, kognitivno jezikoslovje

\section{ABSTRACT}

\section{Synaesthetic metaphors and sound}

This paper analyses a phenomenon called synaesthetic metaphors, in which one sensory modality is described in terms of another (e.g. bright sound, sharp taste, sweet smell). The aim of the research, based on the contrastive analysis of Slovenian and French music reviews, is to determine which sense domains are employed for (synaesthetic) description of sound, more precisely music, and how frequent they are. In this regard the paper examines the directionality principle according to which more concrete and more accessible lower senses (touch, taste, smell) tend to map onto less concrete and less accessible higher senses (sound, sight). Furthermore, the analysis focuses on two other aspects: the conventionality of synaesthetic metaphors and their connotative meaning. Since the source and target domain in synaesthetic transfers belong to sense domains, the paper emphasizes the experiential - i.e. perceptive, cognitive, and sociocultural - dimension of this phenomenon, with regard to which rises the main question concerning the universal and cultural tendencies of synaesthetic metaphors.

Key words: synaesthesia, metaphor, perception, music, sound, cognitive linguistics 